
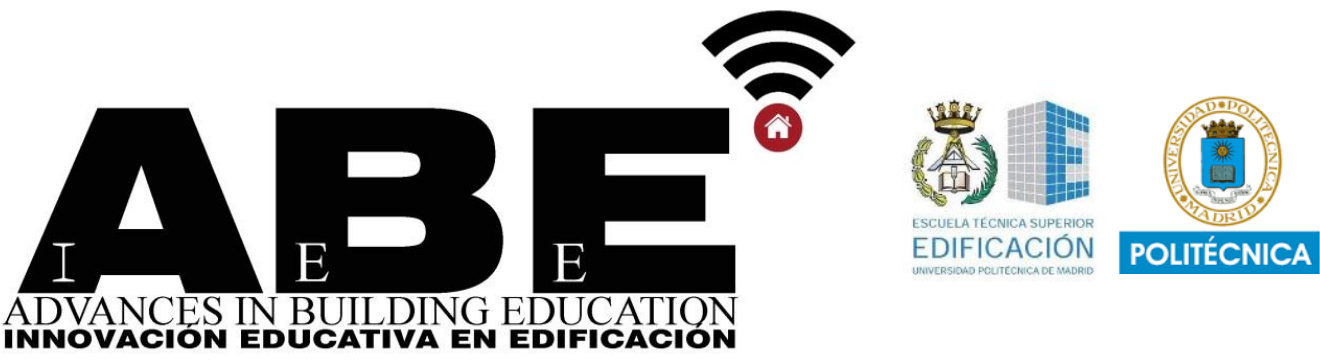

\title{
Estudio de la carga de trabajo percibida y de la calidad docente en los grados de la Escuela Técnica Superior de Arquitectura y Edificación de la Universidad Politécnica de Cartagena.
}

Study of workload and teaching quality of higher technical school for architecture and building degrees in the Universidad Politécnica de Cartagena

\footnotetext{
Vázquez Arenas, G..; García Martín, A. ${ }^{2}$; Conesa Pastor, J.; García León, J. ${ }^{4}$; Solano Fernández, J. P.5

${ }^{1,5}$ Departamento de Arquitectura y Tecnología de la Edificación. Escuela Técnica Superior de Arquitectura y Edificación. Universidad Politécnica de Cartagena. Paseo Alfonso XIII, 52, 30203 Cartagena. España..

${ }^{2}$ Departamento de Ingeniería Minera, Geológica y Cartográfica. Escuela Técnica Superior de Ingeniería de Caminos Canales y Puertos y de Ingeniería de Minas. Universidad Politécnica de Cartagena. España.

${ }^{3}$ Departamento de Expresión Gráfica. Escuela Técnica Superior de Ingeniería Industrial. Universidad Politécnica de Cartagena. España.

${ }^{4}$ Departamento de Electrónica, Tecnología de Computadores y Proyectos. Escuela Técnica Superior de Ingeniería Industrial. Universidad Politécnica de Cartagena. España.

${ }^{6}$ Departamento de Ingeniería Térmica y de Fluidos. Escuela Técnica Superior de Ingeniería Industrial. Universidad Politécnica de Cartagena. España.

* Corresponding author email: gemma.vazquez@upct.es
}

\section{Recibido: 14/07/2017 | Aceptado: 18/10/2017 | Fecha de publicación: 31/08/2018}

DOI: $10.20868 / a b e .2018 .2 .3788$

\section{TITULARES}

- Existen indicadores suficientemente fiables para cuantificar la calidad docente.

- Los resultados del análisis permiten rebatir algunos mitos bastante extendidos.

- Los estudiantes saben distinguir la docencia de calidad y tienen criterio para valorarla.

- La carga de trabajo no influye en la satisfacción con la actividad docente.

\section{HIGHLIGHTS}

- Reliable indicators are sufficient to quantify teacher quality.

- Analysis results allow us to refute some widespread myths.

- Analysis results allow us to refute some widespread myths.

- The workload does not influence the satisfaction with the teaching activity.

Advances in Building Education / Innovación Educativa en Edificación ｜ ISSN: 2530-7940 | http://polired.upm.es/index.php/abe

| Cod. 0026 | Mayo - Agosto 2018 | Vol. 2. № 2 | pp. 45/62 | 
Estudio de la carga de trabajo percibida y de la calidad docente en los grados de la Escuela Técnica Superior de Arquitectura y Edificación de la Universidad Politécnica de Cartagena.

Gemma Vázquez Arenas, Antonio García Martín, Julián Conesa Pastor, Josefina García León, Juan Pedro Solano Fernández

\section{RESUMEN}

La Universidad Politécnica de Cartagena está cuantificando, desde el curso 2014-2015, la carga de trabajo percibida por sus estudiantes. Para ello se emplea una escala Likert en la que los estudiantes evalúan la carga de trabajo de cada una de las asignaturas que cursan pudiendo incluir, si lo desean, comentarios en el cuestionario. Por otra parte, se realizan encuestas de satisfacción con la actividad docente desde hace años. El principal objetivo de este trabajo es determinar si la satisfacción de los estudiantes con la actividad docente del profesorado está relacionada con la carga de trabajo y con los resultados académicos de cada asignatura, es decir si son los profesores menos exigentes los que, en general, obtienen mejores resultados en las encuestas de satisfacción. El estudio se basa en los valores obtenidos en las encuestas de carga de trabajo y de satisfacción y en los resultados académicos del curso 2014-2015 para las asignaturas obligatorias de los Grados en Arquitectura y en Ingeniería de Edificación de la Escuela Técnica Superior de Arquitectura y Edificación de la Universidad Politécnica de Cartagena. La principal conclusión es que existe muy poca relación entre las variables estudiadas y que los resultados de las encuestas de calidad pueden considerarse fiables y robustos.

Palabras clave: Carga de trabajo percibida; Satisfacción con la actividad docente; Resultados académicos; Coordinación horizontal.

\section{ABSTRACT}

Since the academic year 2014-2015, Polytechnic University of Cartagena is quantifying the workload perceived by their students. This is used a Likert scale in which students evaluate the workload of each of the subjects enrolled and can include comments on the questionnaire. On the other hand, satisfaction with the teaching activity surveys are carried out for years. The main objective of this study is to determine if students' satisfaction with the teaching activity is related to workload and academic results of each subject, to be precise, i.e. if are less demanding teachers in general, get better results in the satisfaction surveys. The study is based on the values obtained in the perceived workload, students' evaluation of teaching quality and academic results of the course 2014-2015 for the subjects of the degree in Architecture and Building Engineering of the Higher Technical School of Architecture and Building of the Polytechnic University of Cartagena. The main conclusion is that there is very little relationship between the studied variables and also results of students' evaluation of teaching quality can be considered firm and reliable.

Keywords: Perceived workload; Satisfaction with teaching activity; Academic results; Horizontal coordination.

Advances in Building Education / Innovación Educativa en Edificación | ISSN: 2530-7940 | http://polired.upm.es/index.php/abe

| Cod. 0026 | Mayo - Agosto 2018 | Vol. 2. № 2 | pp. 45/62 | 


\section{INTRODUCCIÓN}

\subsection{Las encuestas de satisfacción con la actividad docente}

Las encuestas de satisfacción de los estudiantes con la actividad docente desarrollada por el profesorado son habituales en las universidades españolas y extranjeras. Forman parte de los modelos de evaluación de la actividad docente y de los sistemas de garantía de calidad de los títulos, y se consideran uno de los indicadores de calidad de las universidades [1]. En ellas se pide a los estudiantes que valoren diferentes aspectos de la actividad docente, que pueden variar de unas universidades a otras. Los cuestionarios empleados suelen incluir una pregunta para evaluar globalmente la docencia desarrollada por cada profesor. La Universidad Politécnica de Cartagena (UPCT) emplea un cuestionario de 11 preguntas, la última de las cuales es "En términos generales estoy satisfecho con la actividad docente desarrollada por el profesor".

La valoración se hace mediante una escala Likert entre 1 y 5 , de forma que los valores altos indican una elevada satisfacción. Un valor expresado en una escala de este tipo puede transformarse a una escala entre 0 y 10 , restándole una unidad y multiplicando el resultado por 2,5. Así, el valor central de la escala Likert (3) equivale al 5 de la escala decimal.

La evaluación de la actividad docente por parte de los estudiantes es un tema controvertido sobre el que existen distintas opiniones y bastantes publicaciones, la mayor parte de ellas procedentes de los países anglosajones. Se han publicado varias revisiones de la literatura existente sobre la calidad de la docencia universitaria y las formas de evaluarla. Algunos estudios cuestionan distintos aspectos de las evaluaciones de la actividad docente ("SETs") en EEUU y Canadá, aunque también existen estudios a favor [2-3]. En general se considera que la evaluación de la actividad docente es fundamental, aunque, según algunos, la metodología que suele emplearse debería ser revisada y mejorada. Hay autores que cuestionan las encuestas de satisfacción porque, en su opinión, estas muestran sesgos, y hay otros que las defienden y afirman que los estudiantes son perfectamente capaces de evaluar la docencia [4]. Sea como sea, uno de los aspectos más controvertidos es el de la publicación de los resultados de las encuestas, que, como es natural, los estudiantes ven de forma mucho más positiva que los profesores.

La actividad docente es muy reconocida en los discursos oficiales, pero apenas se considera en los procesos de acreditación y evaluación del profesorado universitario [5]. Algunas universidades tienen en cuenta, para la promoción de su profesorado, los resultados de la valoración de los estudiantes, pero muchas no lo hacen. La realización de las encuestas de satisfacción con la actividad docente solo tiene sentido si se usan sus resultados para mejorar la calidad docente [6].

\subsection{La carga de trabajo del estudiante y su cuantificación}

Entre los distintos aspectos que conforman el contexto académico, la carga de trabajo es uno de los que en mayor medida condicionan el comportamiento académico de los estudiantes, ya que incide directamente en el tipo de enfoque que estos adoptan frente a su propio aprendizaje e influye de forma significativa en sus resultados académicos. La dedicación real que requiere del estudiante una actividad docente $\mathrm{o}$ una asignatura debería coincidir sensiblemente con la carga prevista para esa actividad o esa asignatura en la memoria de verificación del título y en la guía docente, pero no siempre 
sucede así. Una carga de trabajo superior a la prevista en una o varias asignaturas puede afectar al rendimiento del estudiante y favorecer que adopte un enfoque superficial frente al aprendizaje.

La influencia de la carga de trabajo y las consecuencias que puede provocar un exceso, o una distribución inapropiada, de la misma han sido descritas en diversos estudios [7-13]. Se advierte en ellos que una demanda excesiva de trabajo puede ser contraproducente, pues tiene implicaciones negativas en la calidad del aprendizaje y potencia el abandono de los estudios [14]. Los excesos de carga dificultan la coordinación horizontal, al impedir que el trabajo del estudiante se distribuya de forma adecuada a lo largo de cada cuatrimestre [15].

La carga de trabajo prevista se emplea en el diseño de los planes de estudio y se refiere al número de créditos ECTS, o a su equivalente en horas, que se ha establecido para cada asignatura. En las guías docentes figura también la carga prevista desglosada por actividades, es decir el número de horas que se supone que el estudiante tendrá que dedicar a cada actividad para alcanzar los resultados del aprendizaje propuestos. El tiempo que emplean distintos estudiantes para realizar una misma tarea puede ser muy distinto; por eso se entiende que la carga de trabajo considerada es la que se refiere a lo que se denomina "estudiante medio".

La carga de trabajo real es el número de créditos, o de horas, que un estudiante necesita realmente para completar con éxito una actividad docente o una asignatura. La carga de trabajo real resulta difícil de medir con precisión, ya que para hacerlo se requiere una recogida de información diaria o, al menos, semanal, y hay autores que ponen en duda la fiabilidad de los datos aportados por los estudiantes [9]. Contribuye a ello la gran variabilidad de los valores obtenidos. La UPCT realizó en 2013-
2014 una experiencia piloto en la que pudieron comprobarse tanto las dificultades que supone la cuantificación de la carga de trabajo real como las grandes diferencias entre los valores declarados por distintos estudiantes para una misma actividad [16].

La experiencia piloto sirvió también para poner a prueba un procedimiento para cuantificar la carga de trabajo percibida. Esta no se mide en horas o en créditos ECTS, como las cargas prevista y real, sino mediante variables cualitativas que pueden transformarse en valores numéricos cuando sea preciso. Su interés radica en que es la percepción del contexto académico, y no el propio contexto, lo que condiciona el aprendizaje: muchos autores defienden que es la carga percibida, y no la real, la que influye en el comportamiento académico del estudiante $y$, por tanto, la que debe considerarse y medirse [8], [11], [17]. Se ha comprobado que la carga percibida es un constructo complejo que puede estar influenciado por un amplio rango de aspectos interrelacionados de la enseñanza y el aprendizaje [12]. De hecho, es posible conseguir que los estudiantes trabajen mucho y bien, sin que perciban una carga excesiva, creando un entorno de aprendizaje como el descrito en [11].

La carga de trabajo percibida se cuantifica en la UPCT mediante cuestionarios que se pasan, cada cuatrimestre, en las asignaturas obligatorias de todos los títulos de Grado y de Máster habilitante [16]. El estudiante valora de una vez la carga de todas las asignaturas que cursa, lo que le lleva a compararlas, a establecer diferencias entre ellas y a incorporar matizaciones; de este modo mejora, en nuestra opinión, su validez. Se emplea una escala Likert con valores que van desde 1 ("carga muy baja") a 5 ("carga excesiva"). La figura 1 muestra un ejemplo de cuestionario, del Grado en Ingeniería de Edificación. 
Estudio de la carga de trabajo percibida y de la calidad docente en los grados de la Escuela Técnica Superior de Arquitectura y Edificación de la Universidad Politécnica de Cartagena. Gemma Vázquez Arenas, Antonio García Martín, Julián Conesa Pastor, Josefina García León, Juan Pedro Solano Fernández

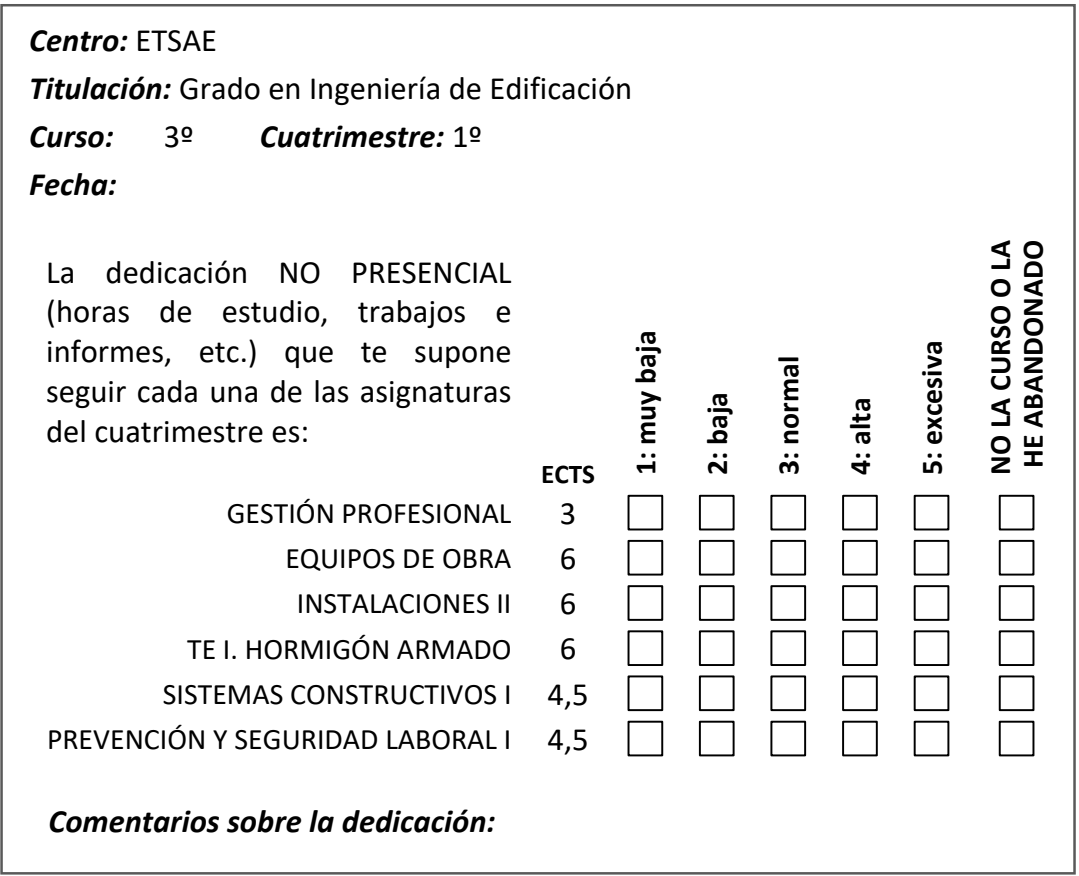

Fig. 1. Uno de los cuestionarios empleados para cuantificar la carga de trabajo percibida.

El valor central de la escala (3) corresponde a aquellas asignaturas que, en opinión de los estudiantes encuestados, requieren una carga de trabajo no presencial similar a la prevista. Se consideran anómalos los valores iguales 0 superiores a 4 y los iguales o inferiores a 2. Las encuestas de carga de trabajo percibida se pasan unas semanas antes del final de cada cuatrimestre, antes del periodo de exámenes y en la misma época que las de satisfacción, aunque de forma independiente a estas. Los resultados de la encuesta se envían a los Centros para que actúen en los casos de asignaturas con valores anómalos, como paso previo indispensable para que pueda realizarse una coordinación horizontal efectiva de los títulos. Los valores más elevados, superiores a 4,5 , identifican casos en los que la carga puede afectar también al rendimiento de los estudiantes, mientras que los valores muy bajos indicarían cargas de trabajo que no permitirán alcanzar los objetivos recogidos en el plan de estudios.

\subsection{Los resultados académicos}

Los resultados académicos nos servirán para caracterizar y cuantificar la "dificultad" de cada una de las asignaturas implicadas en el estudio. De entre los indicadores disponibles nos limitaremos a considerar los tres siguientes:

- tasa de presentados: es el porcentaje de estudiantes presentados a examen frente al de matriculados.

- tasa de éxito: es el porcentaje de estudiantes que han superado el examen sobre el de presentados.

- tasa de rendimiento: es el porcentaje de estudiantes que han superado el examen sobre el de matriculados.

Los valores de estos tres indicadores están relacionados entre sí: por ejemplo, es fácil deducir que la tasa de rendimiento es el producto de las otras dos tasas. Los valores bajos de las tasas corresponderían a las asignaturas más exigentes o de mayor dificultad. 


\subsection{Objetivos}

Existen opiniones 0 creencias bastante extendidas en lo que se refiere a la evaluación de la actividad docente por los estudiantes [18]. Entre esas opiniones está la de los que consideran que los estudiantes no están, en general, capacitados para evaluar a sus profesores y que esas evaluaciones contienen sesgos inaceptables. Según otra de esas opiniones, los estudiantes tienden a valorar mejor a los profesores que menos les exigen y a los que imparten las asignaturas más fáciles de aprobar y que presentan mejores resultados académicos. De acuerdo con esa opinión, los profesores que hacen trabajar a sus estudiantes y se muestran razonablemente exigentes con ellos obtendrían resultados mediocres o malos en las encuestas de satisfacción.

Existen estudios que concluyen que una baja carga de trabajo y unos buenos resultados académicos no implican mejores resultados en las encuestas [19], aunque en asignaturas que requieran un gran esfuerzo de los estudiantes para superarlas, este esfuerzo sí podría influir. Algunos autores sostienen que la carga de trabajo está poco relacionada con la valoración que hacen los estudiantes de la labor del profesorado [16]. Otros autores defienden que los estudiantes son, pese a ciertos mitos y opiniones, capaces de identificar y valorar la docencia de calidad [20]. Si la evaluación de la actividad docente presenta sesgos es, según este autor, porque no se han usado adecuadamente los instrumentos de evaluación.

El principal objetivo de este trabajo es analizar si los resultados de las encuestas de satisfacción de los estudiantes con la actividad docente pueden estar condicionados por el esfuerzo que representa el seguimiento de cada asignatura o por las expectativas previas de una buena calificación. Es decir, pretendemos determinar si existe o no una relación clara entre los resultados de las encuestas de satisfacción y los de la carga de trabajo, y entre los de las encuestas de satisfacción y los resultados académicos. El estudio se centrará en las titulaciones de Grado de la Escuela Técnica Superior de Arquitectura y Edificación (ETSAE) de la UPCT: Grado en Arquitectura y Grado en Ingeniería de Edificación. Puesto que disponemos de datos de todos los títulos de Grado y Máster habilitante de la UPCT, los valores obtenidos para los títulos de Edificación pueden compararse con los de otros Centros de esta Universidad y situarse en su contexto.

La docencia de muchas de las asignaturas de los dos títulos de Grado de la ETSAE se imparte en dos grupos separados. Aprovecharemos esta circunstancia para comprobar la fiabilidad de los datos de satisfacción y de carga de trabajo, comparando los valores obtenidos en los dos grupos de cada asignatura. Esto puede aportarnos una información adicional muy valiosa sobre la validez de los métodos de cuantificación que estamos empleando.

Nuestra hipótesis es que los estudiantes suelen responder con rigor a las encuestas de satisfacción, incluso aunque duden de la utilidad de estas encuestas. Además, saben distinguir la docencia de calidad y la valoran, de forma que en su evaluación tienen poca influencia la carga de trabajo y los resultados académicos.

La actividad docente tiene actualmente una presencia mínima en los programas de acreditación del profesorado universitario. Según se intenta transmitir por parte de los responsables de ANECA, la única razón es que resulta muy difícil medir la calidad de la docencia, lo que no sucede con la investigación y con la gestión. Con este trabajo se quiere demostrar que, por el contrario, existen indicadores suficientemente fiables para cuantificar la calidad docente y destacar su importancia. 
Estudio de la carga de trabajo percibida y de la calidad docente en los grados de la Escuela Técnica Superior de Arquitectura y Edificación de la Universidad Politécnica de Cartagena. Gemma Vázquez Arenas, Antonio García Martín, Julián Conesa Pastor, Josefina García León, Juan Pedro Solano Fernández

\section{METOdOLOGÍA}

En los apartados siguientes emplearemos los siguientes acrónimos para las variables en estudio:

- CT: carga de trabajo percibida; escala Likert de 1 a 5.

- S: satisfacción con la actividad docente; escala Likert de 1 a 5.

- TP: tasa de presentados; valores entre 0 y 100 $\%$.

- TE: tasa de éxito; valores entre 0 y $100 \%$.

- TR: tasa de rendimiento; valores entre 0 y 100 $\%$.

Además, emplearemos los acrónimos GA y GIDE para los Grados en Arquitectura y en Ingeniería de Edificación, respectivamente.

\subsection{Método de ensayo}

Este trabajo se basa en los datos de satisfacción, carga de trabajo y tasas de presentados, éxito y rendimiento, correspondientes al curso 2014-2015, de los títulos de Grado de la ETSAE. Durante el curso 2015-2016 se cambió el plan de estudios del Grado en Arquitectura al de Grado en Fundamentos de Arquitectura. Este cambio supuso la cuatrimestralización de la mayor parte de las asignaturas, que antes eran anuales, y se hizo de una sola vez, no de modo progresivo.

Los valores de satisfacción de los estudiantes con la actividad docente que hemos empleado son los de la pregunta 11 del cuestionario, que evalúa globalmente la actividad docente del profesor.

La unidad de trabajo es la asignatura. Puesto que los datos de carga de trabajo se toman cada cuatrimestre, las asignaturas anuales tienen dos valores, uno por cuatrimestre; para esa variable se ha empleado, en esos casos, la media de ambos valores. Las encuestas de satisfacción se hacen de forma independiente para todos los profesores de cada asignatura; por eso, en asignaturas con más de un profesor, se ha empleado para esa variable la media de los valores individuales. Por otra parte, las asignaturas de los cursos 2 ㅇ y 3 o del GIDE y de los cursos 1으 a 3 ㅇ del GA se impartieron en dos grupos. Cada grupo se ha considerado como si fuese una asignatura independiente, ya que puede haber diferencias en el profesorado, en la organización docente, en la carga de trabajo percibida, etc.

Nuestra base de datos incluye los valores de las variables $C T, S, T P, T E$ y TR de las asignaturas obligatorias de los Grados GA y GIDE. Disponemos además de los datos de las asignaturas obligatorias de títulos de Grado y de Máster habilitante de la UPCT. No todas las asignaturas disponen de toda la información; el número de datos de cada variable se muestra en la tabla 1.

\begin{tabular}{|c|c|c|c|c|c|}
\cline { 2 - 6 } \multicolumn{1}{c|}{} & CT & S & TP & TE & TR \\
\hline GIDE & 64 & 62 & 62 & 62 & 62 \\
\hline GA & 51 & 51 & 46 & 46 & 46 \\
\hline Total ETSAE & 115 & 113 & 108 & 108 & 108 \\
\hline Total UPCT & 888 & 864 & 882 & 882 & 882 \\
\hline
\end{tabular}

Tabla 1. Número de datos de cada variable.

La mayoría de las asignaturas del GA, 27 sobre 31, eran anuales en 2014-2015. Por el contrario, solo 4 de las 41 asignaturas del GIDE eran anuales. Las asignaturas impartidas en dos grupos fueron 24 en el GIDE y 20 en el GA. 
Estudio de la carga de trabajo percibida y de la calidad docente en los grados de la

Escuela Técnica Superior de Arquitectura y Edificación de la Universidad Politécnica de Cartagena.

Gemma Vázquez Arenas, Antonio García Martín, Julián Conesa Pastor, Josefina García León, Juan Pedro Solano Fernández

\subsection{Los métodos}

Nuestro análisis se apoya en herramientas estadísticas sencillas. Se han calculado los estadísticos elementales de todas las variables, para el conjunto de los datos y desagregados por Centros, cursos, etc. Los valores correspondientes a los Grados de la ETSAE se han comparado entre sí y con los del resto de la UPCT. Se han trazado y comparado los histogramas de las distintas variables.

Las posibles relaciones entre variables, especialmente $S$ con CT y S con TP, TE y TR, se han estudiando mediante el coeficiente de correlación de Pearson y se han visualizado mediante gráficos de correlación. Recordamos que el coeficiente de correlación mide el grado de intensidad de la relación lineal entre dos variables cuantitativas. Su valor oscila entre -1 y +1 , indicando si la posible relación es negativa, es decir, que una variable aumenta en la medida que la otra disminuye, o positiva. La interpretación de los coeficientes de correlación ( $\rho)$ se hará de forma simplificada, empleando la escala de la tabla 2.

\begin{tabular}{cc}
\hline Valor de $\boldsymbol{\rho}$ & Significado \\
\hline-1 a $-0,7$ & Correlación negativa fuerte \\
\hline$-0,7$ a $-0,4$ & Correlación negativa moderada \\
\hline$-0,4$ a $-0,2$ & Correlación negativa débil \\
\hline$-0,2$ a 0 & Correlación negativa muy débil o nula \\
0 a 0,2 & Correlación positiva muy débil o nula \\
\hline 0,2 a 0,4 & Correlación positiva débil \\
\hline 0,4 a 0,7 & Correlación positiva moderada \\
0,7 a 1 & Correlación positiva fuerte \\
\hline
\end{tabular}

Tabla 2. Escala empleada para interpretar los coeficientes de correlación.
La comparación de los valores de una variable entre dos grupos de la misma asignatura se ha realizado mediante gráficos de barras $y$ numéricamente, calculando las diferencias y el coeficiente de correlación entre ambas series de valores.

\section{RESULTADOS Y DISCUSIÓN}

\subsection{Valores medios de las variables: Contextualización}

La tabla 3 muestra los valores de las variables CT, S, TR, TE y TP, en la ETSAE y los restantes Centros de la UPCT, para el curso 2014-2015. Los valores medios de la UPCT se han obtenido promediando los de todas las asignaturas, y no a partir de los valores medios por Centros.

\begin{tabular}{lccccc}
\hline \multicolumn{1}{c}{ Centro } & CT & S & TP & TE & TR \\
\hline $\begin{array}{l}\text { ETSI Caminos } \\
\text { Minas }\end{array}$ & 3,42 & 3,77 & 78,5 & 82,0 & 65,4 \\
\hline $\begin{array}{l}\text { ETS Arquitectura y } \\
\text { Edificación }\end{array}$ & 3,42 & 3,59 & 77,3 & 79,8 & 62,6 \\
\hline ETSI Agronómica & 3,54 & 3,63 & 76,2 & 83,5 & 64,3 \\
\hline ETSI Industrial & 3,35 & 3,63 & 78,6 & 77,5 & 62,2 \\
\hline $\begin{array}{l}\text { ETSI Naval } \\
\text { Oceánica }\end{array}$ & 3,47 & 3,61 & 75,3 & 74,9 & 57,8 \\
\hline $\begin{array}{l}\text { ETSI } \\
\text { Telecomunicación }\end{array}$ & 3,39 & 3,60 & 79,9 & 76,9 & 63,4 \\
\hline $\begin{array}{l}\text { F Ciencias de la } \\
\text { Empresa }\end{array}$ & 3,40 & 3,61 & 80,8 & 76,5 & 62,6 \\
\hline Centros adscritos & 3,26 & 3,76 & 94,9 & 96,4 & 91,4 \\
\hline \multicolumn{1}{c}{ Total UPCT } & $\mathbf{3 , 3 8}$ & $\mathbf{3 , 6 5}$ & $\mathbf{8 0 , 7}$ & $\mathbf{8 0 , 8}$ & $\mathbf{6 6 , 5}$ \\
\hline
\end{tabular}

Tabla 3. Valores de CT, S, TP, TE y TR en los distintos Centros de la UPCT, 2014-2015.

Advances in Building Education / Innovación Educativa en Edificación | ISSN: 2530-7940 | http://polired.upm.es/index.php/abe

| Cod. 0026 | Mayo - Agosto 2018 | Vol. 2. № 2 | pp. 45/62 | 
Estudio de la carga de trabajo percibida y de la calidad docente en los grados de la Escuela Técnica Superior de Arquitectura y Edificación de la Universidad Politécnica de Cartagena. Gemma Vázquez Arenas, Antonio García Martín, Julián Conesa Pastor, Josefina García León, Juan Pedro Solano Fernández

Destacan los resultados excepcionales de los Centros adscritos, especialmente en TP, TE y TR. La ETSAE muestra valores intermedios, en general. En $S$ obtiene un valor muy parecido al de la mayoría de los Centros. Los valores de CT son superiores a 3 en todos los Centros, y el valor medio de la UPCT para esta variable es 3,38 .

La figura 2 muestra la distribución de las variables $S$ y $C T$ en la ETSAE. En la figura se aprecia que casi el $40 \%$ del profesorado del Centro obtiene valores de $S$ entre 3,51 y 4,00 . El $80,5 \%$ de los estudiantes declaran una satisfacción mayor de 3 y el 22,1 \% mayor de 4 .
El nivel de satisfacción es, por tanto, razonable en la ETSAE, con solo un $19,5 \%$ de valores inferiores a 3 y un 4,4\% inferiores a 2 .

En la figura 2 se ve que la mayor parte de las asignaturas de la ETSAE obtiene valores de CT entre 2,51 y 4 . Casi un $82 \%$ de las asignaturas se sitúa en lo que consideramos carga normal, con un $17,4 \%$ de valores superiores a 4 y menos del $1 \%$ inferiores a 2 . Recordamos que 4 es el valor por encima del cual se considera que la carga de trabajo de la asignatura es excesiva y 2 es el valor por debajo del cual se considera carga escasa. Estos valores anómalos son los que deben revisar el Centro y los Departamentos responsables de las asignaturas que los obtienen.

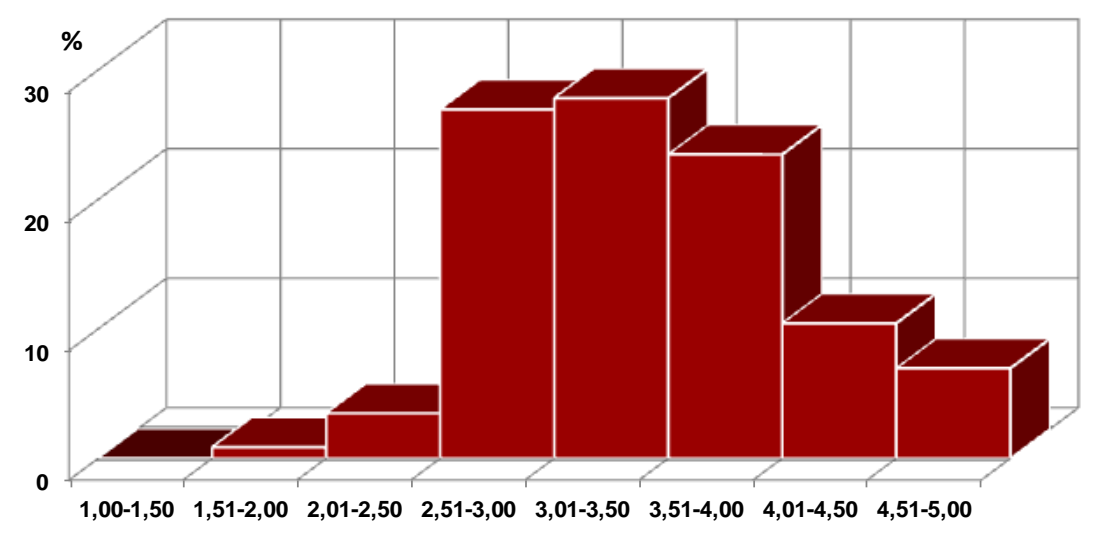

Carga de trabajo (CT)

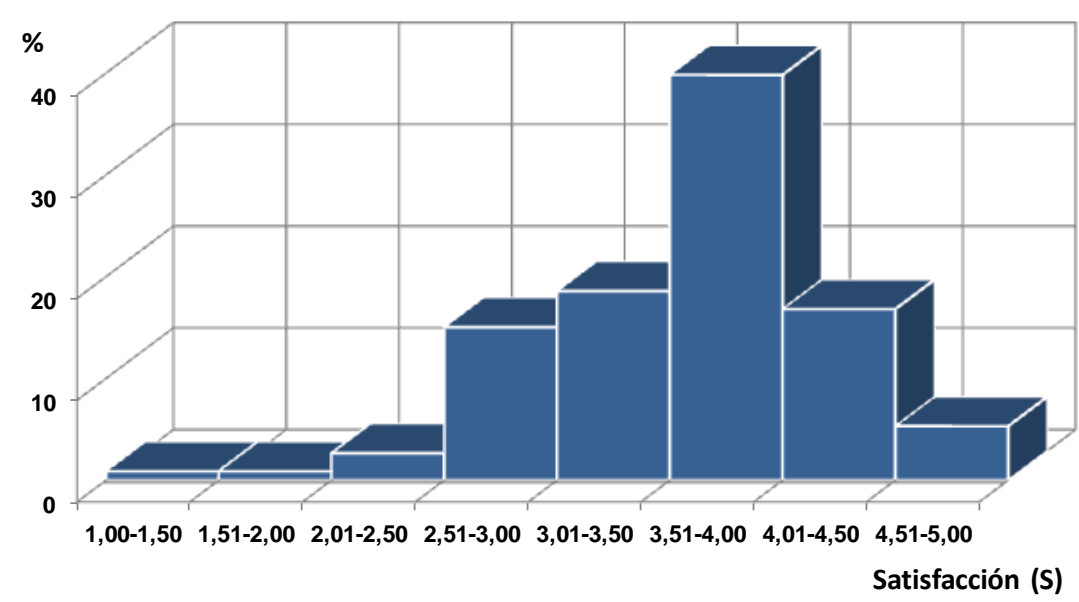

Fig. 2. Histogramas de carga de trabajo y de satisfacción con la actividad docente en la ETSAE. 
Estudio de la carga de trabajo percibida y de la calidad docente en los grados de la Escuela Técnica Superior de Arquitectura y Edificación de la Universidad Politécnica de Cartagena. Gemma Vázquez Arenas, Antonio García Martín, Julián Conesa Pastor, Josefina García León, Juan Pedro Solano Fernández

La tabla 4 muestra los valores medios por cursos de todas las variables, en GIDE y GA. Los valores más bajos de CT se obtienen, en ambos títulos, en primer curso, mientras que los más altos se obtienen en cuarto curso. Esto es lo habitual en la UPCT y puede deberse a que las asignaturas básicas de los primeros cursos son las que menos emplean las metodologías docentes participativas y la evaluación continua, que para muchos estudiantes parecen suponer una mayor carga de trabajo. Otra posible causa es que muchos estudiantes pasan de curso con asignaturas pendientes de cursos anteriores y esta situación, acumulativa conforme se progresa en el título, puede contribuir a que, de forma general, se vaya incrementando su percepción de la carga de trabajo.

\begin{tabular}{cccccc}
\hline \multicolumn{6}{c}{ GIDE } \\
\hline Curso & CT & S & TP & TE & TR \\
1ㅇ & 3,14 & 3,48 & 68,48 & 69,95 & 48,29 \\
2o & 3,54 & 3,63 & 77,03 & 85,00 & 66,03 \\
3ㅇ & 3,50 & 3,65 & 79,31 & 74,97 & 60,40 \\
4ㅇ & 3,73 & 3,08 & 83,09 & 76,48 & 64,34 \\
\hline & \multicolumn{5}{c}{ GA } \\
Curso & CT & S & TP & TE & TR \\
1ㅇ & 3,12 & 3,48 & 71,33 & 69,60 & 49,68 \\
2ㅇ & 3,47 & 3,65 & 77,91 & 84,07 & 66,39 \\
3ㅇ & 3,48 & 3,66 & 77,22 & 78,00 & 60,92 \\
4ㅇ & 3,64 & 3,34 & 80,38 & 80,48 & 65,18 \\
5o & 3,25 & 3,86 & 88,35 & 90,12 & 80,19 \\
\hline
\end{tabular}

Tabla 4. Valores de CT, S, TP, TE y TR por curso en GIDE y GA, 2014-2015.

Respecto a S, los dos títulos muestran el mismo comportamiento entre los cursos primero y cuarto: los valores más bajos se obtienen en cuarto curso, mientras que los cursos segundo y tercero muestran valores similares a la media de la UPCT. En quinto curso del GA se obtiene un valor de 3,86, que muestra una alta satisfacción con la actividad docente. Los valores bajos de CT de primer curso no coinciden con valores altos de $S$ (3,48 en ambos títulos); sin embargo, los valores más altos de $\mathrm{CT}$, en cuarto curso, sí coinciden con los más bajos de $\mathrm{S}$. El mejor valor de $\mathrm{S}$, el de quinto curso del GA $(3,86)$, coincide con un valor de CT relativamente bajo $(3,25)$.

Los valores de TP, TE y TR crecen, en general aunque no siempre, a medida que progresa el título. Los valores de TP alcanzan valores de $83,09 \%$, en cuarto curso del GIDE, y de 88,35 $\%$, en quinto curso del GA. Los valores de primer curso son bajos, debido a que en ese curso es obligatoria la matrícula de todas las asignaturas para los estudiantes a tiempo completo; eso no sucede en los restantes cursos. Esa circunstancia afecta a los valores de todas las tasas.

El comportamiento de las cinco variables estudiadas sigue en la ETSAE pautas similares a las que se observan en la mayoría de los restantes Centros de la UPCT.

\subsection{Resultados por grupos}

Muchas de las asignaturas de la ETSAE se imparten en dos grupos diferentes. Los dos grupos de cada asignatura deben trabajar con la misma metodología docente y con el mismo sistema de evaluación, es decir con la misma guía docente. No obstante puede haber diferencias entre ellos, por ejemplo en el profesorado que los imparte o en la organización (horarios de mañana o de tarde), y estas diferencias podrían reflejarse en los valores de las variables en estudio.

Además, pueden existir dudas respecto a la validez de los valores de las variables $\mathrm{S}$ y $\mathrm{CT}$, ya que ambas se obtienen a partir de encuestas que rellenan los estudiantes [18]. Para intentar 
Estudio de la carga de trabajo percibida y de la calidad docente en los grados de la Escuela Técnica Superior de Arquitectura y Edificación de la Universidad Politécnica de Cartagena. Gemma Vázquez Arenas, Antonio García Martín, Julián Conesa Pastor, Josefina García León, Juan Pedro Solano Fernández

disiparlas hemos comparado los resultados de las variables entre los grupos $A$ y $B$ de cada una de las 44 asignaturas de la ETSAE en las que la docencia está repartida. Si los resultados entre grupos fuesen muy dispares cabría pensar que los estudiantes no rellenan las encuestas con rigor, o que el azar contribuye de forma excesiva. En ambos casos, la validez de los datos podría quedar en entredicho.

Los valores de CT son similares en los dos grupos de la mayoría de las asignaturas. Solo hay una asignatura en la que la diferencia entre ellos es mayor de $1(1,25)$. Las diferencias puntuales no significan que se esté empleando una metodología distinta, sino que pueden deberse a la forma en que la aplica el profesorado de cada grupo o, simplemente, deberse a cuestiones organizativas. En cualquier caso, son poco importantes, por lo que no vamos a detenernos a analizarlas.

La mayoría de las asignaturas también muestran, en general, valores similares de $S$ aunque, en este caso, hay cuatro asignaturas que superan la diferencia de 1. Dos de ellas, una de cada título, presentan diferencias muy grandes en esta variable: 2,52 y 3,19. Estas diferencias significan que uno de los grupos obtiene un valor alto de $S$ y el otro un valor anormalmente bajo, inferior a 2. Pueden deberse a que el profesorado que imparte la asignatura en los dos grupos no es el mismo, pero también podrían deberse a que en uno de los grupos se ha producido un problema, o un desacuerdo, entre los estudiantes y el profesor que ha afectado al valor de S.

Las figuras $3 a$ y $3 b$ muestran los valores de Satisfacción (S) y Carga de Trabajo (CT), a modo de ejemplo, en tercer curso del GIDE; la figura 4 muestra los de tercer curso del GA. Para CT se han resaltado los valores límite de lo que consideramos carga anómala por exceso y por defecto. Se aprecia en ambas figuras que los valores de CT son similares en los dos grupos de cada asignatura, con diferencias que no suelen superar el $10 \%$. En S se aprecian, para varias asignaturas del GIDE (figura 3a), diferencias algo mayores, aunque todas inferiores a 1; para el GA las diferencias son muy pequeñas (figuras $4 a$ y $4 b$ ).

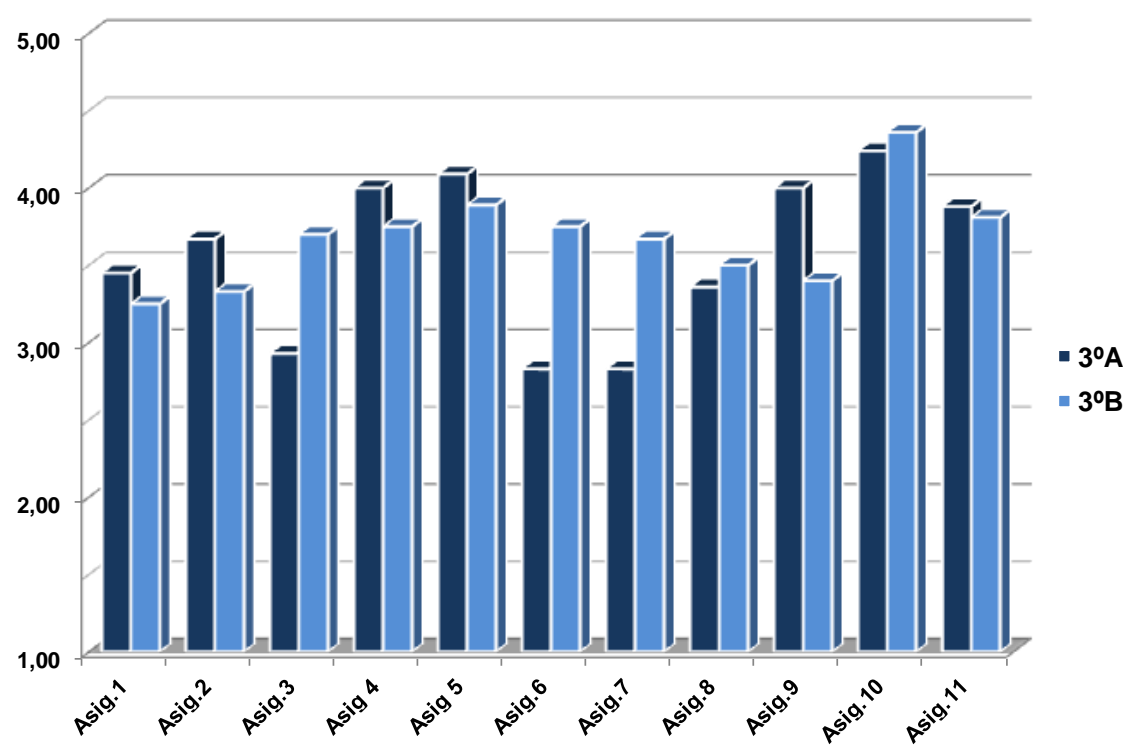

Fig. 3a. Satisfacción por grupos. Tercer curso del GIDE. 
Estudio de la carga de trabajo percibida y de la calidad docente en los grados de la Escuela Técnica Superior de Arquitectura y Edificación de la Universidad Politécnica de Cartagena.

Gemma Vázquez Arenas, Antonio García Martín, Julián Conesa Pastor, Josefina García León, Juan Pedro Solano Fernández

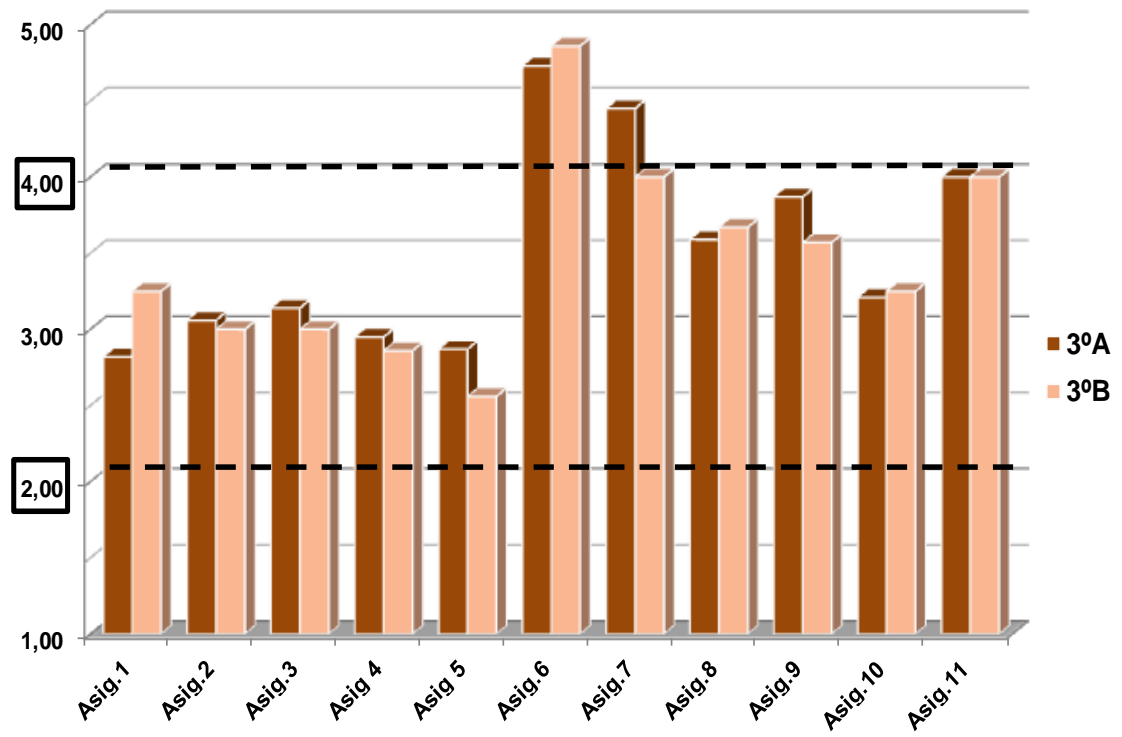

Fig. 3b. Carga de trabajo por grupos. Tercer curso del GIDE.

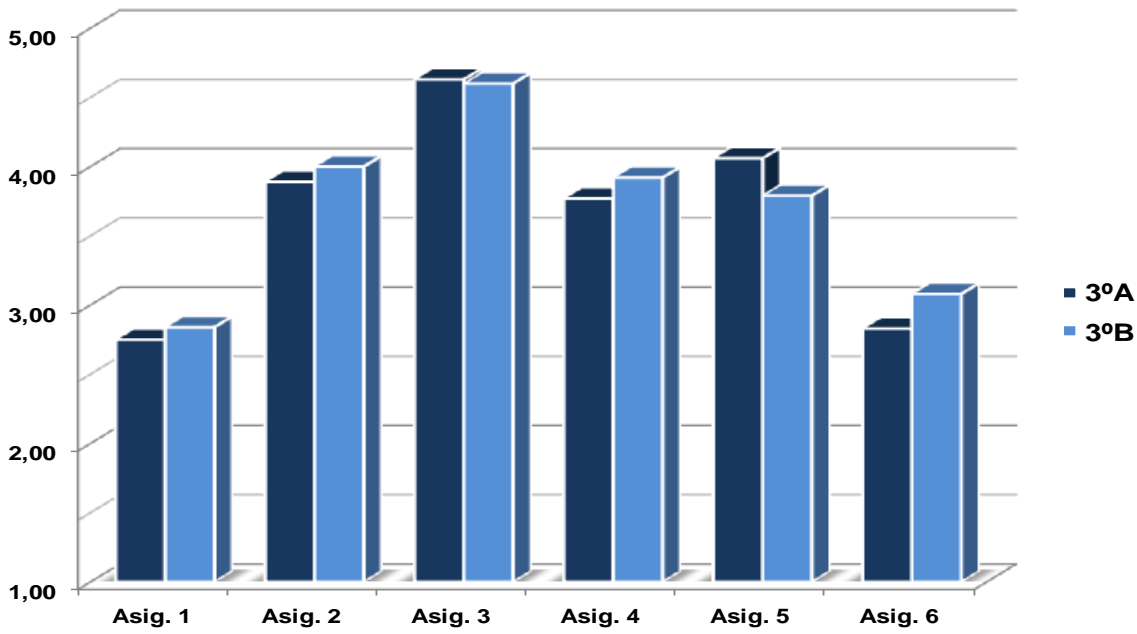

Fig. 4a. Satisfacción por grupos. Tercer curso del GA.

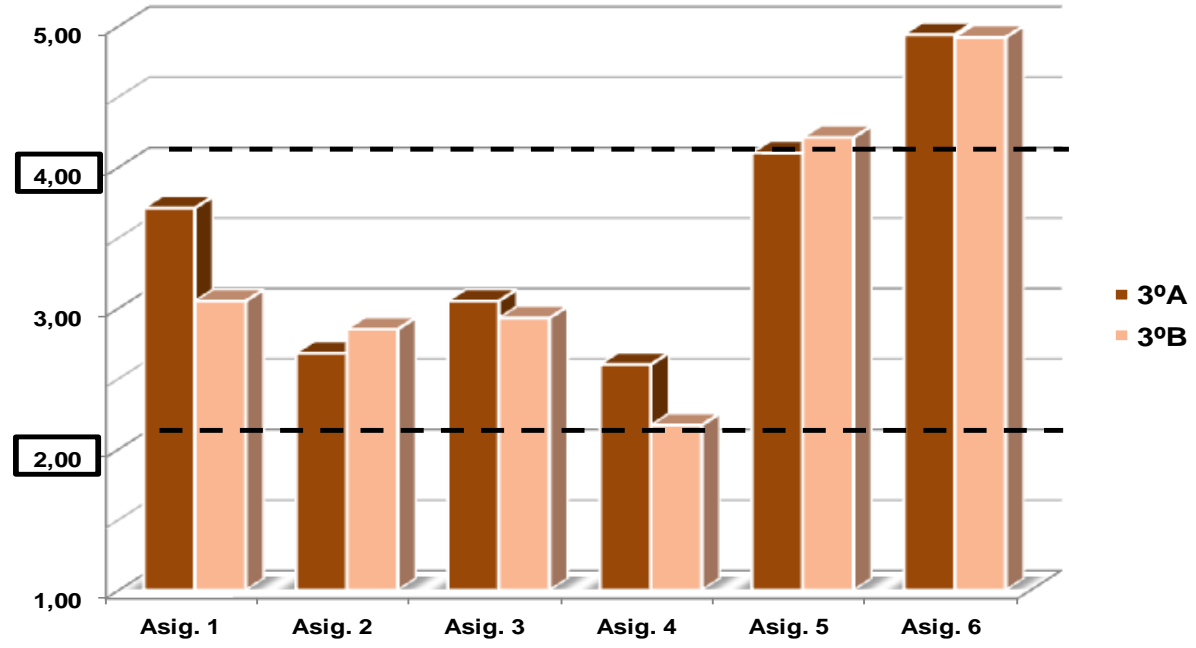

Fig. 4b. Carga de trabajo por grupos. Tercer curso del GA.

Advances in Building Education / Innovación Educativa en Edificación ｜ ISSN: 2530-7940 | http://polired.upm.es/index.php/abe

| Cod. 0026 | Mayo - Agosto 2018 | Vol. 2. № 2 | pp. 45/62 | 
Se ha comprobado que la situación es parecida en otros títulos de la UPCT para las asignaturas que se imparten en dos grupos: la mayoría de los valores son similares, pero se dan, puntualmente, diferencias que pueden llegar a ser importantes.

Estas diferencias grandes entre grupos, que se dan de forma esporádica tanto en CT como en $\mathrm{S}$, deben ser analizadas por los responsables de los títulos, especialmente si coinciden con valores anómalos, para determinar sus posibles causas.

\subsection{Comentarios en las encuestas de CT de la ETSAE}

Los estudiantes que rellenan las encuestas pueden incluir, si lo desean, comentarios. Hemos revisado los de la encuesta de carga de trabajo percibida de la ETSAE para conocer las causas de los valores anómalos de CT.

La mayor parte de los comentarios hace referencia a asignaturas concretas, cuya carga se percibe como excesiva, y es frecuente que sean varios los estudiantes que comentan cada una de esos casos. Se comprueba fácilmente que los comentarios de este tipo casi siempre corresponden a las asignaturas con mayores valores de CT.

Muchos comentarios se limitan a indicar que la dedicación que requiere la asignatura es excesiva, o que es mayor que la de otras asignaturas con mayor carga prevista, sin aportar más detalles; otros indican cuál es la causa del exceso de carga percibida, por ejemplo, demasiados trabajos.

Unos pocos comentarios hacen referencia a la poca relación que existe entre la dedicación de alguna asignatura y los resultados académicos de los estudiantes que la cursan. Según ellos, en estas asignaturas un mayor esfuerzo del estudiante no se refleja en una mejor calificación, y lo que se está cuestionando es la metodología de evaluación por entender que no es del todo justa. En otro caso se citan dificultades concretas para realizar unas tutorías que son esenciales para aprobar el trabajo anual de la asignatura.

Unos pocos comentarios se refieren a la organización del plan de estudios, indicando que hay cursos en los que se concentran las asignaturas con más carga. En realidad, todos los cursos tienen la misma carga prevista (60 créditos ECTS) pero, como sabemos, no la misma carga percibida, que es la variable que se debe ajustar. En ese sentido, uno de los estudiantes comenta que el tercer curso del GIDE es el que tiene una mejor distribución de la carga de trabajo. En la tabla 4 se comprueba que este curso presenta un buen equilibrio entre estas variables, con un valor de CT intermedio $(3,50)$ y el mejor valor de $S(3,65)$ de esta titulación

Uno de los comentarios del GA es una queja sobre las asignaturas anuales. Como hemos indicado, el cambio de plan de estudios introducido en 2015-2016 ha supuesto la transformación de la mayor parte de ellas en cuatrimestrales.

Alguno de los comentarios se refiere a la organización de los horarios. En opinión de los estudiantes que los han escrito, las actividades presenciales están organizadas de manera que no se facilita el trabajo no presencial.

Otros estudiantes comentan la mala distribución de la carga y la deficiente organización de las actividades en algunos cuatrimestres, debida a la falta de coordinación. Los procedimientos de coordinación horizontal ya se han implantado de forma sistemática en la ETSAE, lo que debe eliminar los problemas de este tipo. 
Estudio de la carga de trabajo percibida y de la calidad docente en los grados de la Escuela Técnica Superior de Arquitectura y Edificación de la Universidad Politécnica de Cartagena. Gemma Vázquez Arenas, Antonio García Martín, Julián Conesa Pastor, Josefina García León, Juan Pedro Solano Fernández

\subsection{Análisis de correlación}

Si la carga de trabajo influye en la satisfacción con la actividad docente, la relación entre S y CT debería ser de signo negativo, de forma que los valores altos de CT supondrían valores bajos de $S$ y viceversa. En cuanto a las tasas, si estas influyen en S, la relación entre S y TP, TE y TR debería ser positiva, ya que los buenos resultados académicos supondrían valores altos de S. La relación entre CT y las tasas debería ser negativa, de forma que una mayor carga conlleve peores resultados académicos.

La tabla 5 muestra los valores del coeficiente de correlación ( $\rho$ ) entre $S$ y las restantes variables en estudio y entre CT y las restantes variables, para los dos Grados de la ETSAE y para el conjunto de la UPCT. El número de datos en cada caso figura en la tabla 1.

\begin{tabular}{ccccc}
\hline S con & CT & TP & TE & TR \\
\hline GIDE & $-0,05$ & 0,21 & 0,03 & 0,17 \\
\hline GA & $-0,17$ & 0,23 & 0,26 & 0,26 \\
\hline Total ETSAE & $-0,12$ & 0,21 & 0,11 & 0,20 \\
\hline Total UPCT & $\mathbf{- 0 , 1 1}$ & $\mathbf{0 , 1 4}$ & $\mathbf{0 , 1 9}$ & $\mathbf{0 , 1 9}$ \\
\hline
\end{tabular}

\begin{tabular}{ccccc}
\hline CT con & S & TP & TE & TR \\
\hline GIDE & $-0,05$ & $-0,03$ & - & $-0,26$ \\
\hline GA & $-0,17$ & $-0,19$ & 0,16 & 0,01 \\
\hline Total ETSAE & $-0,12$ & $-0,09$ & - & $-0,14$ \\
\hline Total UPCT & $-\mathbf{0 , 1 1}$ & $-\mathbf{0 , 2 1}$ & - & - \\
\hline & & & $\mathbf{0 , 1 5}$ & $\mathbf{0 , 2 1}$ \\
\hline
\end{tabular}

Tabla 5. Valores del coeficiente de correlación $\rho$ entre $S y$ las restantes variables y entre CT y las restantes variables.
Todos los valores del coeficiente de correlación son, según la escala recogida en la tabla 2, débiles o muy débiles/nulos. Los correspondientes a $\mathrm{S}$ tienen los signos que cabía esperar: negativo con CT y positivos con TP, TE y TR. Los valores correspondientes a CT tienen los valores que cabía esperar salvo en las tasas TE y TR del GA, para las que se observan correlaciones positivas. En cualquier caso, se trata de valores muy reducidos, que muestran una influencia débil de las variables CT, TP, TE y TR en la satisfacción de los estudiantes y de CT en las variables TP, TE y TR.

La escasa correlación entre CT y S puede verse de forma gráfica en el diagrama de dispersión de la figura 5. Se han superpuesto en el mismo diagrama los datos de la ETSAE, en rojo, y los del conjunto de la UPCT, en azul.

Cuando existe una fuerte correlación lineal, los puntos se distribuyen siguiendo, aproximadamente, una línea recta. Sin embargo, la nube que aparece en la figura 5 no muestra ninguna forma ni dirección preferente. Esto ocurre tanto para los datos de la ETSAE como para el conjunto de la UPCT.

Es interesante señalar que hay asignaturas con CT superior a 4 y que presentan también un valor alto de $S$, aunque no hay ninguna que haya obtenido un 5 en ambas variables. Por otra parte, la única asignatura con un valor de 1 en $S$, lo que supone una satisfacción mínima, obtiene también un 1 en CT. Esa asignatura, que no se imparte en la ETSAE, se aleja mucho de los valores medios en la UPCT.

También se han calculado los valores del coeficiente de correlación por cursos, para los títulos de la ETSAE y para los de la UPCT. En algunos de ellos pueden observarse valores más altos del coeficiente, pero siempre para un número de datos muy reducido y, por tanto, poco representativo. 
Estudio de la carga de trabajo percibida y de la calidad docente en los grados de la Escuela Técnica Superior de Arquitectura y Edificación de la Universidad Politécnica de Cartagena. Gemma Vázquez Arenas, Antonio García Martín, Julián Conesa Pastor, Josefina García León, Juan Pedro Solano Fernández

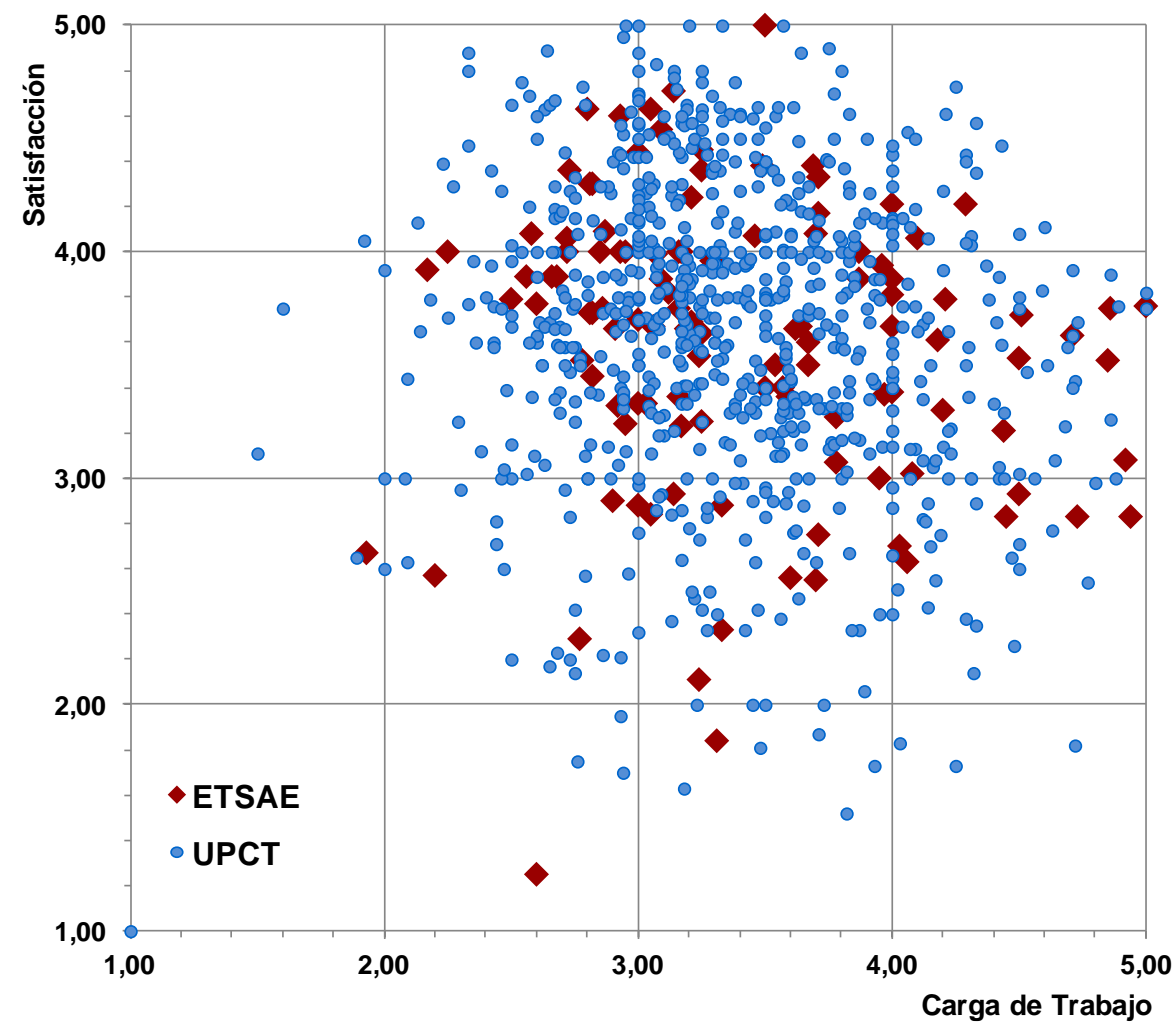

Fig. 5. Satisfacción por grupos. Tercer curso del GA.

\section{CONCLUSIONES}

Las conclusiones que podemos extraer del presente estudio están alineadas con las que han expresado otros autores de distintos países y ámbitos académicos. Los resultados obtenidos para la Escuela Técnica Superior de Arquitectura y Edificación (ETSAE) son coherentes con los del resto de la Universidad de la que forma parte, que oferta principalmente títulos de las ramas de Ingeniería y Arquitectura y de Ciencias Sociales y Jurídicas. Las conclusiones son, por tanto, válidas para toda la UPCT. El estudio se ha basado en el análisis de las relaciones de las variables "carga de trabajo percibida" y "tasas de resultados académicos" con la variable "satisfacción de los estudiantes con la actividad docente". La carga de trabajo percibida es una estimación de la dedicación media que cada una de las asignaturas requiere del estudiante que las cursa; las tasas de resultados académicos representan la dificultad media que supone cada una de esas asignaturas.

Los resultados del análisis nos permiten rebatir algunos mitos bastante extendidos en el campo de la evaluación de la calidad de la actividad docente por parte de los estudiantes universitarios. Según estos mitos, los estudiantes no responden a las encuestas con rigor, no son capaces de evaluar la calidad docente y valoran peor a los profesores que se muestran razonablemente exigentes con ellos o que imparten asignaturas cuyos resultados académicos no son muy altos.

El análisis comparativo de los valores de carga de trabajo percibida obtenidos por los dos grupos en que se imparte buena parte de las asignaturas de la ETSAE muestra que, en general, estos valores son similares, sin grandes diferencias. Respecto a los resultados de las 
encuestas de satisfacción, se dan también valores similares aunque, puntualmente, puedan encontrarse diferencias significativas. Lo mismo sucede en otras asignaturas de la UPCT que se imparten en grupos independientes. La conclusión es que los estudiantes rellenan las encuestas con el debido rigor, de manera que las mismas condiciones en el desarrollo de la actividad docente provocan similares resultados en las encuestas. Estos resultados demuestran ser, por tanto, fiables y robustos.

Del análisis de los datos cualitativos de las encuestas de carga de trabajo percibida se deduce que los comentarios expresados por los estudiantes son coherentes con los valores medios obtenidos por las asignaturas; es decir, las asignaturas con cargas excesivas son las que tienen los comentarios negativos. También se recogen comentarios sobre los horarios, la coordinación horizontal y vertical, la metodología docente y de evaluación, etc. Los comentarios positivos corresponden a cursos con un buen equilibrio de carga de trabajo y valores razonables de satisfacción. Esto contribuye a dar validez a esos valores.

Ha quedado demostrada la escasa correlación entre las variables "satisfacción" y "carga de trabajo percibida" y entre las variables "satisfacción" y "tasas de resultados académicos", tanto para las dos titulaciones de la ETSAE como para el conjunto de la UPCT, ya que en todos los casos se han observado correlaciones débiles o nulas. La conclusión es que los profesores que hacen trabajar a los estudiantes y se muestran exigentes con ellos, siempre dentro de lo razonable, pueden obtener magníficos resultados en la encuesta de satisfacción. También se comprueba que una carga de trabajo escasa y unos resultados académicos altos no garantizan un valor elevado en la encuesta de satisfacción. En consecuencia, la influencia de la carga y de la dificultad de la asignatura en la valoración de su profesorado es muy reducida.

En el diagrama de dispersión de la figura 5 se observa que la mayor concentración de asignaturas se produce en la zona comprendida entre los valores 2,5 y 4 de carga de trabajo y entre los valores 3 y 5 de satisfacción. Esa es la zona de carga de trabajo normal y de satisfacción media-alta. Las cargas de trabajo muy elevadas, superiores a 4,5, corresponden a valores de satisfacción que nunca superan el 4 . Además, los datos cualitativos de las encuestas suelen referirse a esas asignaturas. Nuestra conclusión, también alineada con los de otros autores, es que cuando la carga de trabajo se percibe como excesivamente alta sí puede influir en la satisfacción del estudiante. Esa percepción tiene que ver con la dedicación excesiva, pero también con otros aspectos del entorno de aprendizaje, como son los siguientes: actividades docentes sin utilidad aparente, métodos de evaluación poco relacionados con los resultados del aprendizaje previstos, escasa disponibilidad del profesorado, etc.

Por todo ello concluimos que la carga de trabajo y los resultados académicos influyen muy poco en la satisfacción que muestran los estudiantes con la actividad docente. De acuerdo con los resultados del estudio, los estudiantes demuestran ser capaces de evaluar la calidad docente sin dejarse influir de manera importante por aspectos como la dificultad de la asignatura o su nivel de exigencia.

Los estudiantes saben distinguir la docencia de calidad y tienen criterio para valorarla. Por lo tanto, la calidad docente se puede evaluar y debe tenerse en cuenta en la carrera profesional de los docentes. La falta de consideración de la actividad docente por parte de ANECA, justificada por una supuesta imposibilidad de medirla con cierta precisión, puede contribuir a impedir que esta adquiera la importancia que 
Estudio de la carga de trabajo percibida y de la calidad docente en los grados de la Escuela Técnica Superior de Arquitectura y Edificación de la Universidad Politécnica de Cartagena. Gemma Vázquez Arenas, Antonio García Martín, Julián Conesa Pastor, Josefina García León, Juan Pedro Solano Fernández

sabemos que tiene e ir en detrimento de la calidad de nuestras universidades.

\section{REFERENCIAS}

[1] Marsh H. Students' evaluations of university teaching: dimensionality, reliability, validity, potential biases and usefulness. Perry RP and Smart JC (eds.). The Scholarship of Teaching and Learning in Higher Education: An EvidenceBased Perspective, 2007, pp. 319-383.

[2] Ramírez MI, Montoya J. La evaluación de la calidad de la docencia en la universidad: Una revisión de la literatura. Revista de Docencia Universitaria, 2014, vol. 12, pp. 77-95.

[3] Pounder J. Is student evaluation of teaching worthwhile? An analytical framework for answering the question. Quality Assurance in Education, 2007, vol. 15 (2), pp. 178-191.

[4] Sánchez F, Rubio R, Alonso E, Retamal K. La valoración de la actividad docente. Algo más que la opinión de los estudiantes. Boletín de Psicología, 2009, № 97, pp. 71-92.

[5] Mas O. Las competencias del docente universitario: la percepción del alumno, de los expertos y del propio protagonista. Revista de Docencia Universitaria, 2012, vol. 10 (2), pp. 299318.

[6] Álvarez V, García E, Gil J. La calidad de la enseñanza universitaria desde la perspectiva de los profesores mejor valorados por los alumnos. Revista de Educación, 1999, vol. 319, pp. 273290.

[7] Trigwell K, Prosser M. Improving the quality of student learning: The influence of learning context and student approaches to learning on learning outcomes. Higher Education, 1991, vol. 22, pp. 251-266.
[8] Ramsden P. Learning to teach in higher education. London: Kogan Page, 1992.

[9] Chambers E. Work-load and the quality of student learning. Studies in Higher Education, 1992, vol. 17 (2), pp. 141-153.

[10] Lizzio A, Wilson K, Simons R. University students' perceptions of the learning environment and academic outcomes: implications for theory and practice. Studies in Higher Education, 2002, vol. 27(1), pp. 27-52.

[11] Kember D, Leung DYP. Characterising a teaching and learning environment conducive to making demands on students while not making their workload excessive. Studies in Higher Education, 2006, vol. 31(2), pp. 185-198.

[12] Giles L. An investigation of the relationship between students' perceptions of workload and their approaches to learning at a regional polytechnic. Doctoral Thesis. Massey University, New Zealand, 2009.

[13] Biggs J, Tang C. Teaching for quality learning at university (4 ${ }^{\mathrm{a}}$ ed.). Buckingham: The Society for Research into Higher Education \& Open University Press, 2011.

[14] Lockwood F. Estimating student workload, readability and implications for student learning and progression. In: 17th ODLAA Conference: Breaking the Boundaries: The International Experience in Open, Distance and Flexible Education, Adelaide, Australia, 2005.

[15] García Martín A, Andreu MM, Briones AJ, Busquier S, Conesa J, García Cascales MS, García-León J, García del Toro MA, GarcíaAyllón S, Herrero R, Maciá JF, Martínez M, Mendoza A, de Miguel MD, Peñalver MJ, Periago P, Ros D, Serrano JL, Solano JP, Tomás 
Estudio de la carga de trabajo percibida y de la calidad docente en los grados de la Escuela Técnica Superior de Arquitectura y Edificación de la Universidad Politécnica de Cartagena. Gemma Vázquez Arenas, Antonio García Martín, Julián Conesa Pastor, Josefina García León, Juan Pedro Solano Fernández

A. Coordinación docente horizontal y vertical.

CRAI UPCT ediciones, 2015.

[16] Herrero R, García Martín A. Equipos Docentes: experiencias y resultados (20132014). CRAI UPCT ediciones, 2014, pp. 3-67. ISBN: 978-48-16325-01-6.

[17] Dee KC. Student perceptions of high course workload are not associated with poor student evaluations of instructor performance. Journal of Engineering Education, 2007, pp. 69-78.

[18] Marsh H, Roche L. Effects of grading leniency and low workload on students' evaluations of teaching: popular myth, bias, validity, or innocent bystanders? Journal of Educational Psychology, 2000, Vol. 92(1), pp. 202-228.

[19] Thornton B, Adams M, Sepehri M. The impact of students' expectations of grades and perceptions of course difficulty, workload, and pace on faculty evaluations. Contemporary Issues in Education Research, 2010, Vol. 3(12), pp. 1-6.

[20] Ramsden P. A performance indicator of teaching quality in higher education: the experience questionnaire. Studies in Higher Education, 1991, vol. 16, pp. 129-150. 